\title{
O QUE SÃO FRACTAIS: UMA ANÁLISE CONCEITUAL DE SUAS CARACTERÍSTICAS A PARTIR DE PESQUISAS BRASILEIRAS
}

\author{
WHAT ARE FRACTALS: A CONCEPTUAL ANALYSIS OF ITS \\ CHARACTERISTICS FROM BRAZILIAN RESEARCH
}

Fabio Antunes Brun de Campos ${ }^{1}$

ORCID iD: 0000-0001-7614-392X

Minéia Cappellari Fagundes ${ }^{2}$

ORCID iD: $\underline{0000-0002-9016-1128}$

\begin{abstract}
RESUMO
Este artigo apresenta uma pesquisa de abordagem qualitativa, de cunho documental, na qual buscamos investigar o que são fractais e quais são as características que os definem. A motivação para este artigo surgiu no contexto de uma pesquisa de mestrado envolvendo o ensino da matemática com tais estruturas geométricas. Nessa pesquisa, durante a leitura de alguns trabalhos científicos, percebemos divergências teóricas em relação às características que definem os fractais. Isso instigou-nos a aprofundar os nossos conhecimentos sobre esse conceito e a buscarmos alternativas que contribuíssem com a comunidade científica e com os processos de ensino da matemática com fractais. Dessa forma, pesquisamos, na Biblioteca Digital Brasileira de Teses e Dissertações (BDTD), trabalhos que versassem sobre o ensino da matemática com fractais e analisamos as definições nelas apresentadas por meio da metodologia da Análise Textual Discursiva. As características encontradas nos 36 trabalhos analisados foram: a irregularidade, a autossemelhança, a complexidade infinita e a dimensão fracionária. Assim, este artigo apresenta indícios de conceitos sobre os fractais que podem contribuir para o desenvolvimento científico e educacional sobre este tema.
\end{abstract}

Palavras-chave: Características dos fractais. Definição de fractal. Ensino da matemática.

\begin{abstract}
This article presents a research of a qualitative approach, of documental nature, in which we aim to investigate what fractals are and what the characteristics that define them are. The motivation for this article arose in the context of a master's research involving the teaching of Mathematics with such geometric structures. In this research, while reading some scientific papers, we noticed theoretical divergences in relation to the characteristics that define fractals. This prompted us to deepen our knowledge of this concept and to look for alternatives that could contribute to the scientific community and to the processes of teaching Mathematics with fractals. Therefore, we researched, in the Biblioteca Digital Brasileira de Teses e Dissertações (BDTD), works that dealt with the teaching of Mathematics with fractals and analyzed the definitions presented in them through the Discursive Textual Analysis method. The characteristics found in the 36 studies analyzed were: irregularity, self-similarity, infinite complexity and fractal dimension. Therefore, this article presents evidence of concepts about fractals that can contribute to scientific and educational development on this topic.
\end{abstract}

\footnotetext{
${ }^{1}$ Mestre em Ensino de Ciências e Matemática pelo Programa de Pós-Graduação em Ensino de Ciências e Matemática (PPGECM). Atualmente, é docente da Universidade do Estado de Mato Grosso (Unemat), Barra do Bugres, Mato Grosso, Brasil.E-mail: fabio.campos@unemat.br.

${ }^{2}$ Doutora em Engenharia Elétrica pela Universidade Estadual Paulista (Unesp), docente do Programa de Programa de Pós-Graduação em Ensino de Ciências e Matemática (PPGECM) da Universidade do Estado de Mato Grosso (Unemat), Barra do Bugres, Mato Grosso, Brasil.E-mail: mineia@unemat.br.
} 
Keywords: Characteristics of fractals. Definition of fractal. Mathematics teaching.

\section{INTRODUÇÃO}

A motivação para este artigo surgiu no contexto de uma revisão de literatura desenvolvida em uma pesquisa de mestrado realizada na Universidade do Estado de Mato Grosso (Unemat), campus de Barra do Bugres. Nessa revisão, percebemos algumas dificuldades, por parte de pesquisadores da área da educação e do ensino da matemática, na proposição de uma definição para o termo "fractal". Nas pesquisas então encontradas, deparamo-nos com categorias de termos semelhantes, as quais poderiam contribuir para uma caracterização dos fractais. Isso instigou-nos, à época, a buscar respostas para as questões: o que são fractais e quais são as características que os definem? A partir disso, surgiu este artigo, que visa discorrer sobre essas questões.

Várias pesquisas científicas têm se dedicado ao estudo dos fractais, tanto as de cunho educacional, com ênfase na educação matemática, quanto aquelas que envolvem outras áreas das ciências (NEGRI, 2014; BARBOSA, 2019). Na verdade, as aplicações dessas estruturas geométricas nas ciências, fora do contexto educacional, têm sido uma das justificativas para a inserção delas no contexto educacional (BAIER, 2005). No que se refere à abordagem dos fractais na Educação Básica, de acordo com Baier (2005, p. 137), é possível articular “o currículo da Matemática tanto com o mundo dos alunos como também com o mundo da ciência”, abrangendo conteúdos matemáticos do Ensino Fundamental e do Ensino Médio. Segundo Luz (2016, p. 50), no Ensino Fundamental, “é possível estudar conceitos da geometria Euclidiana, como área e perímetro de figuras planas e regularidades”, e, no Ensino Médio, abordando tópicos mais variados, “é possível trabalhar área e perímetro, progressão aritmética e geométrica e probabilidade geométrica”, entre outros conceitos.

Em se tratando de aplicações nas ciências, os fractais têm contribuído para o estudo de temas variados. Entre eles, podemos citar: desordem na atmosfera; variação populacional de espécies; observações das oscilações do coração e do cérebro; interligações microscópicas de vasos sanguíneos nas ramificações alveolares; cotações de bolsas comerciais de valores; desenvolvimento de estruturas arquitetônicas; e ambientes digitais para jogos e filmes (BARBOSA, 2005; BAPTISTA, 2013; CAMPOS; FAGUNDES, 2018).

De acordo com Barbosa (2005, p. 19), a formulação de um "conceito de fractal ainda tem muito a desejar", principalmente no que se refere a algo que sirva e caiba apenas a essa 
estrutura. Andrade Júnior (2015) comenta também que “conceituar fractais não é algo simples e as tentativas para definir tal conceito têm deixado algumas lacunas" em relação à formulação das características que definem essas figuras geométricas. A partir disso, entendemos que pesquisar os termos usados para a definição de fractais no contexto das pesquisas atuais sobre o ensino da matemática pode indicar caminhos para o processo de caracterização da geometria envolvida nesse assunto e, consequentemente, contribuir para as pesquisas científicas na área e para os processos de ensino e aprendizagem da matemática.

Desse modo, neste artigo, buscamos investigar o que são fractais e quais são as características que os definem, por meio da análise de 36 trabalhos brasileiros, publicados nos últimos 15 anos, encontrados na Biblioteca Digital Brasileira de Teses e Dissertações (BDTD). Nesses trabalhos, a irregularidade, a autosemelhança, a complexidade infinita e a dimensão fracionária foram identificadas como características que definem essas estruturas. Com base nisso, esperamos que o artigo possa se tornar um ponto de partida para novas compreensões, discussões e dimensionamentos sobre o que são fractais e quais são as características que os definem.

\section{CARACTERÍSTICA DOS FRACTAIS}

Os fractais vêm sendo estudados por vários matemáticos desde o século $\mathrm{XV}$, tais como Dürer (1471-1528), Cantor (1845-1918), Peano (1858-1932), Hilbert (1862-1945), Koch (1870 -1924) e Sierpinski (1882-1969), e os estudos continuam na atualidade (NUNES, 2006; RABAY, 2013). Apesar de os trabalhos matemáticos anteriores ao século XX reconhecerem a existência dos fractais, muitos deles consideravam essas formas como "casos patológicos" ou "monstros matemáticos", indicando que a comunidade matemática desse período não conseguia definir o que eram tais estruturas (MANDELBROT, 1977).

Os avanços teóricos a respeito da Geometria Fractal são atribuídos a Benoit Mandelbrot, matemático francês que viveu entre 1924 e 2010 e que designou esses supostos "monstros matemáticos" de "fractais" (NEGRI, 2014; VIEIRA, 2018). Segundo Mandelbrot (1977, p. 4), esse termo foi "tomado do adjetivo latino fractus", que significa "quebrado, fracionado ou fragmentado". Em relação à definição de "fractais", Mandelbrot afirma que encontrou dificuldades para formular um conceito definitivo, mas resolveu definir essas figuras da seguinte forma: “- Um fractal é, por definição, um conjunto para o qual a dimensão HausdorffBesicovitch excede estritamente a dimensão topológica; - Todo conjunto $\mathrm{D}$, com dimensão 
fracionária é um fractal” (MANDELBROT, 1977, p. 15, tradução nossa). Portanto, o autor considera a dimensão fracionária ou a dimensão de Hausdorff como a principal característica para uma definição dos fractais.

Embora também considere essa dimensão, Falconer (1985) comenta que a tentativa de definição de Mendelbrot "é insatisfatória, pois exclui certos conjuntos de fractais altamente irregulares que claramente devem ser considerados como sendo da natureza fractal" (FALCONER, 1985, p. IX, tradução nossa). Após Falconer publicar duas importantes obras sobre a dimensão de Hausdorff, uma em 1985 e outra em 1990, Barbosa (2005) sugere que os fractais devem ser entendidos a partir das seguintes caracterizações:

Um conjunto F é fractal se, por exemplo:

- F possui alguma forma de 'autossimilaridade' ainda que aproximada ou estatística;

- A dimensão fractal, definida de alguma forma, é maior que a sua dimensão topológica;

- O conjunto $\mathrm{F}$ pode ser expresso através de um procedimento recursivo ou iterativo. (BARBOSA, 2005, p. 18-19).

Apesar de essa definição apresentada por Falconer (apud BARBOSA, 2005) ser a mais aceita pelos pesquisadores, é possível perceber que o processo de formulação de uma definição dos fractais ainda causa certo desconforto em vários autores. São necessárias, portanto, mais pesquisas em relação a esse aspecto conceitual. Pensando nisso e levando em consideração a presença de categorias recorrente que contribuem para a definição das estruturas em questão, em revisões de literatura, adotamos como hipóteses de características os seguintes aspectos: a irregularidade, a autossemelhança, a complexidade infinita e a dimensão fracionária. Em seguida, discorremos sobre cada uma dessas características.

\subsection{A irregularidade}

Instigado pela necessidade de nomear as estruturas geométricas com características não regulares, compostas por diferentes tamanhos de segmentos de reta, por polígonos ora regulares, ora não regulares, por diferentes tipos de curvas, circunferências, elipses e parábolas, ou ainda pela união de várias destas formas em uma única representação, Mandelbrot (1977, p. 5) comenta que "fractal" foi o termo escolhido por ele para designar esses "monstros matemáticos", esses "casos patológicos" que a comunidade matemática desse período não conseguia definir o que eram. Explicando como se deu o processo de sua pesquisa para dar um nome a esses conjuntos geométricos, o matemático francês comenta que associar o termo 
“irregularidade” a essas formas "é sensato, assim como adequado" (MANDELBROT, 1977, p. 5), pois, assim, intuitivamente já estamos nos referindo a uma qualidade evidente nas estruturas em questão.

Sobre esse aspecto irregular dos fractais, Gouvea (2005) comenta que "a palavra 'fractal' tem origem da palavra latina 'fractus', que significa quebrado, irregular'. Percebe-se que Gouvea (2005), da mesma forma como propõe Mandelbrot (1977), usa o termo "fractal" para indicar que a característica de irregularidade faz parte dessas estruturas. Similarmente, Rabay (2013, p. 2) explica que a palavra fractal surge do "adjetivo 'fractus', do verbo 'frangere' em latim, significando partir em segmentos irregulares". Rabay (2013) apresenta a irregularidade como uma das características que definem os fractais. Para esse autor (2013, p. 3), tal característica pode ser entendida como uma "não-suavidade ou fragmentação". No mesmo sentido, para Dalpiaz (2016, p. 24), “os fractais apresentam uma forma geométrica complexa, definida por propriedades como autossimilaridade, irregularidade (forma rugosa ou fragmentada) e uma dimensão não inteira". Esse autor também inclui a irregularidade como uma característica ("propriedade") que define as estruturas em questão.

Ao analisar a categoria "irregularidade" a partir desses autores, indagamos se ela não abrange significados mais profundos do que simplesmente ser um adjetivo ou sinônimo de “fractal". Permitindo ser compreendida como um elemento, uma característica que contribui para compor, explicar e distinguir a estrutura fractal das demais formas geométricas.

\subsection{A autossemelhança e a complexidade infinita}

A autossemelhança e a complexidade infinita foram outras categorias que buscamos investigar como possíveis integrantes da definição dos fractais. Como expresso anteriormente, Falconer inclui a autossemelhança ("autossimilaridade") e a complexidade infinita em sua definição, já Mandelbrot, não considera estas características como parte constituinte da definição dessas estruturas. De todo modo, acreditamos que se faz necessário descrever o que sejam a autossemelhança e a complexidade infinita para que, assim, ao se tentar definir os fractais, essas características sejam conhecidas e mencionadas nos contextos de pesquisa ou ensino.

De acordo com Gouvea (2005, p. 48), denomina-se de "autossimilaridade" a propriedade que uma estrutura geométrica tem de manter seu formato, independentemente da ampliação. Segundo Nunes (2006, p. 29), “uma figura é autossemelhante se apresenta sempre 
o mesmo aspecto visual a qualquer escala que seja ampliada ou reduzida, ou seja, se parte de uma figura se assemelha à figura vista como um todo". Com base nessas citações, percebe-se que os fractais apresentam características de semelhança geométrica relacionadas tanto com os seus lados quanto com os seus ângulos - ambos devem se repetir ao longo de sua estrutura. Por esse motivo, utilizamos o termo "autossemelhaça", em vez de "autossimilaridade", como adotado em várias pesquisas.

Já a complexidade infinita, de acordo Faria (2012, p. 35), “está associada às infinitas iterações que ocorrem na construção de um fractal, pois ele é regido por um padrão que repete sua estrutura própria por uma quantidade ilimitada de vezes". Segundo Nunes (2006, p. 31), tal característica ocorre por meio de "processos recursivos aplicados indefinidamente [ou seja, infinitamente]. Quanto maior for o número de iterações deste processo, mais detalhes serão apresentados e, assim, nunca obteremos uma imagem final”.

\subsection{A dimensão fractal}

Retomemos a definição para os fractais utilizada por Mandelbrot: “- Um fractal é, por definição, um conjunto para o qual, a dimensão Hausdorff-Besicovitch, excede estritamente a dimensão topológica; - Todo conjunto D, com dimensão fracionária é um fractal" (MANDELBROT, 1977, p.15, tradução nossa). A partir dessa definição, é possível perceber que a dimensão fractal não segue a dimensão topológica, com valores $0,1,2$ e 3; segue, antes, a dimensão fracionária de Hausdorff-Besicovitch, com valores decimais entre 0 e 3 (MANDELBROT, 1977). Segundo Mucheroni (2017),

\footnotetext{
O nome dimensão de Hausdorff vem do matemático alemão Felix Hausdorff (18681942), o qual publicou trabalhos na área de topologia e introduziu a ideia dessa dimensão. Alguns autores também se referem a ela por dimensão de HausdorffBesicovitch, pelo fato de seu desenvolvimento ter recebido grandes contribuições do matemático russo Abram Samoilovitch Besicovitch (1891-1970). Anos mais tarde, o matemático francês Benoit Mandelbrot (1924-2010) retomou os estudos de Hausdorff e Besicovitch em seus trabalhos sobre a Geometria Fractal. (MUCHERONI, 2017, p. $45)$.
}

Segundo Faria (2012, p. 39), para realizar o cálculo da dimensão fractal, pode ser utilizada a relação $n=r^{D}$, em que $n$ representa "o número de peças que um fractal possui (produzido em cada uma das partes do fractal geradas em uma mesma iteração)"; $r$ representa o "fator de aumento (necessário para que cada parte ao ser ampliada se iguale a figura do nível 
anterior)". Além disso, " $r$ " também pode ser representado como $\frac{1}{l}$, onde $l$ representa o tamanho, a medida do lado de uma terminada parte do fractal, e D, a dimensão fractal. Realizando a manipulação algébrica dessa equação é possível chegar à fórmula a seguir:

$$
n=r^{D} \rightarrow \log n=\log r^{D} \rightarrow \log n=D \cdot \log r \rightarrow D=\frac{\log n}{\log r} .
$$

Trata-se de uma das fórmulas mais simples para encontrar a dimensão fractal, sendo também indicada por outros autores além de Faria (2012) - por exemplo, Barbosa (2005), Rabay (2013) e Oliveira (2019).

\section{METODOLOGIA}

A abordagem metodológica adotada neste artigo está baseada em métodos qualitativos. A pesquisa pode ser classificada como documental, visto que a investigação se baseia em arquivos disponibilizados na BDTD. A partir de levantamento nessa plataforma, buscou-se analisar categorias de palavras e discursos textuais sobre o ensino da matemática com fractais.

De acordo com Tuzzo e Braga (2016, p. 142), a abordagem qualitativa "não se apresenta como uma proposta rigorosamente estruturada, permitindo que a imaginação e a criatividade levem os investigadores a proporem trabalhos que explorem novos enfoques". Entre outras características, tal abordagem tenta compreender um fenômeno em sua totalidade, se desvencilhando de ideias preconcebidas, enfatizando a subjetividade como meio de compreender e interpretar as experiências, valendo-se, para isso, de instrumentos como a oralidade, a escrita, imagens e percepções do investigador durante a produção de dados (GERHARDT; SILVEIRA, 2009).

Segundo Lüdke e André (1986, p. 38), a pesquisa documental "pode se constituir numa técnica valiosa de abordagem de dados qualitativos, seja complementando as informações obtidas por outras áreas técnicas, seja desvelando aspectos novos de um tema ou problema". Esse tipo de pesquisa "favorece a observação do processo de maturação ou de evolução de indivíduos, grupos, conceitos, conhecimentos, comportamentos, mentalidades, práticas, entre outros" (SÁ-SILVA; ALMEIDA; GUINDANI, 2009, p. 2).

Sendo assim, a busca por teses e dissertações brasileiras na BDTD que respondessem à problemática desta pesquisa - o que são fractais e quais são as características que os definem foi realizada com os seguintes descritores: com os descritores "fractais" e "ensino", obtivemos 48 resultados; utilizamos também "fractais", "ensino" e "matemática" e obtivemos 39

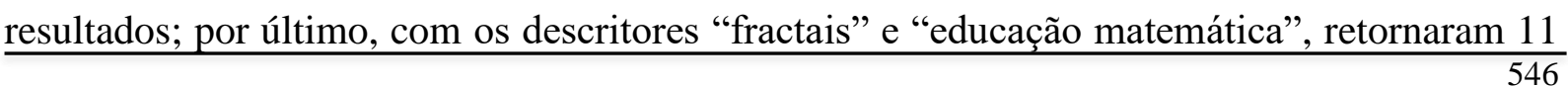
Revista REAMEC, Cuiabá (MT), v. 8, n. 2, p. 540-561, maio-agosto, 2020. 
resultados. De todos esses resultados, decidimos analisar os 39 trabalhos obtidos com os descritores "fractais", "ensino" e "matemática", por entendermos que eles abrangem, de forma concisa, o ensino da matemática com fractais. No quadro 1, listamos 38 trabalhos, pois a dissertação de Santos (2016) foi encontrada em língua portuguesa e em inglês - sendo assim, consideramos apenas a versão no primeiro idioma.

\begin{tabular}{|c|c|c|}
\hline $\begin{array}{l}\text { Autor(a) e ano } \\
\text { de conclusão }\end{array}$ & Título do trabalho & Tipo \\
\hline Eberson (2004) & $\begin{array}{l}\text { "Um estudo sobre a construção de fractais em ambientes computacionais e } \\
\text { suas relações como transformações geométricas no plano" }\end{array}$ & Dissertação \\
\hline Baier (2005) & $\begin{array}{c}\text { "O nexo 'Geometria Fractal - produção da ciência contemporânea' tomado } \\
\text { como núcleo do currículo do Ensino Básico" }\end{array}$ & Tese \\
\hline Gouvea (2005) & $\begin{array}{c}\text { "Um estudo de fractais geométricos através de caleidoscópios e softwares } \\
\text { de geometria dinâmica" }\end{array}$ & Dissertação \\
\hline $\begin{array}{l}\text { Gonçalves } \\
\text { (2007) }\end{array}$ & $\begin{array}{c}\text { "Uma sequência de ensino para o estudo de progressões geométricas via } \\
\text { fractais" }\end{array}$ & Dissertação \\
\hline $\begin{array}{l}\text { Stormowski } \\
\quad(2008)\end{array}$ & “Estudando matrizes a partir de transformações geométricas” & Dissertação \\
\hline $\begin{array}{l}\text { Baldovinotti } \\
\quad(2011)\end{array}$ & $\begin{array}{l}\text { "Um estudo de fractais geométricos na formação de professores } \\
\text { de matemática" }\end{array}$ & Dissertação \\
\hline Vieira (2011) & $\begin{array}{c}\text { "Implicações pedagógicas do lúdico para o ensino e aprendizagem da } \\
\text { álgebra" }\end{array}$ & Dissertação \\
\hline Kindges (2012) & $\begin{array}{l}\text { "Geometria Fractal no Ensino Fundamental: } \\
\text { inserindo matemática contemporânea nos conteúdos do currículo escolar" }\end{array}$ & Dissertação \\
\hline Faria (2012) & $\begin{array}{c}\text { "Padrões fractais: contribuições ao processo de generalização de conteúdos } \\
\text { matemáticos" }\end{array}$ & Dissertação \\
\hline Mineli (2012) & "Fractais: generalização de padrões no Ensino Fundamental" & Dissertação \\
\hline Padilha (2012) & $\begin{array}{c}\text { "Conhecimentos geométricos e algébricos a partir da construção } \\
\text { de fractais com uso do software GeoGebra" }\end{array}$ & Dissertação \\
\hline Adami (2013) & "Fractais no Ensino Médio: uma sequência didática" & Dissertação \\
\hline Brisante (2013) & "Fractais no Ensino Médio" & Dissertação \\
\hline Pereira (2013) & "Fractais circulares: algumas consideraçõ & Dissertação \\
\hline Rabay (2013) & "Estudo e aplicações da Geometria Fractal" & Dissertação \\
\hline $\begin{array}{l}\text { Zambelli } \\
(2013)\end{array}$ & $\begin{array}{c}\text { "Fractais no Ensino Médio: a valorização da geometria a partir de uma } \\
\text { nova experiência em sala de aula" }\end{array}$ & Dissertação \\
\hline Araújo (2014) & "Noções de Geometria Fractal elementar" & Dissertação \\
\hline Freitas (2014) & "Explorando o conjunto de Cantor e outros fractais no Ensino Básico" & Dissertação \\
\hline $\begin{array}{l}\text { Mingoranci } \\
\quad(2014)\end{array}$ & $\begin{array}{c}\text { "A Geometria Fractal aliada à contextualização, protagonismo juvenil e } \\
\text { tecnologias como proposta de melhoria no processo ensino/aprendizagem } \\
\text { da matemática na Educação Básica" }\end{array}$ & Dissertação \\
\hline Moraes (2014) & "Equações de diferenças, caos e fractais" & Dissertação \\
\hline Nicola (2014) & "Conhecendo fractal no Ensino Médio: árvore pitagórica" & Dissertação \\
\hline Paixão (2014) & "O ensino de fractais no Ensino Fundamental" & Dissertação \\
\hline Souza (2014) & "Geometria Fractal e aplicações no Ensino Médio" & Dissertação \\
\hline $\begin{array}{l}\text { Andrade } \\
\text { Júnior (2015) }\end{array}$ & $\begin{array}{l}\text { "Ciência contemporânea a formação de professores: o caso dos fractais em } \\
\text { uma perspectiva Kelliana" }\end{array}$ & Dissertação \\
\hline Araújo (2015) & $\begin{array}{l}\text { "Teoria matemática implícita na Geometria Fractal: construindo fractais } \\
\text { com a ferramenta computacional Asypmtote" }\end{array}$ & Dissertação \\
\hline Modesto (2015) & $\begin{array}{c}\text { "Matemática e arte: explorando a geometria dos fractais e as tesselações de } \\
\text { Escher" }\end{array}$ & Dissertação \\
\hline
\end{tabular}




\begin{tabular}{|c|c|c|}
\hline Luz (2016) & $\begin{array}{c}\text { "A Geometria Fractal como fator minimizador das dificuldades referentes a } \\
\text { conceitos geométricos" }\end{array}$ & Dissertação \\
\hline Santos (2016) & "Circuitos resistivos autossimilares" & Dissertação \\
\hline $\begin{array}{l}\text { Mendes Júnior } \\
\quad \text { (2017) }\end{array}$ & $\begin{array}{l}\text { "Uma nova abordagem dos complexos para o Ensino Médio: o estudo dos } \\
\text { fractais e do caos na composição do conjunto preenchido de Julia e o } \\
\text { conjunto de Mandelbrot" }\end{array}$ & Dissertação \\
\hline Moreira (2017) & "Geometria Fractal na Educação Básica" & Dissertação \\
\hline Santos (2017) & "O misterioso e enigmático mundo de Pascal e Fibonacci" & Dissertação \\
\hline Titoneli (2017) & $\begin{array}{c}\text { "A observação de padrões: modelagem matemática através de sequências } \\
\text { numéricas e objetos geométricos" }\end{array}$ & Dissertação \\
\hline Duarte (2018) & $\begin{array}{c}\text { "Utilização do GeoGebra, de smartphone e de reflexões escritas na } \\
\text { construção de conceitos relacionados a retas paralelas cortadas por uma } \\
\text { transversal" }\end{array}$ & Dissertação \\
\hline $\begin{array}{l}\text { Valmorbida } \\
\text { (2018) }\end{array}$ & $\begin{array}{c}\text { "Uma proposta de atividades para o estudo de progressões geométricas } \\
\text { utilizando fractais e o software GeoGebra" }\end{array}$ & Dissertação \\
\hline Alves (2019) & "Fractais: uma ferramenta no Ensino Médio" & Dissertação \\
\hline Barbosa (2019) & $\begin{array}{c}\text { "Aspetos do pensamento computacional na construção de fractais com } \\
\text { software GeoGebra" }\end{array}$ & Dissertação \\
\hline Oliveira (2019) & "Uma proposta de Geometria de Fractais para a sala de aula" & Dissertação \\
\hline Vieira (2019) & $\begin{array}{c}\text { "O uso da Geometria Fractal como Ferramenta no ensino de progressões } \\
\text { geométricas e logaritmos" }\end{array}$ & Dissertação \\
\hline
\end{tabular}

Ao analisar os trabalhos do Quadro 1, percebemos que o de Vieira (2011) e o de Duarte (2018) não tratavam da temática da nossa investigação - o ensino da matemática com fractais. A dissertação de Vieira (2011) tinha como foco de pesquisa o ensino algébrico da matemática, e a de Duarte (2018), a utilização do software GeoGebra e de smartphones no ensino da matemática. Esses trabalhos, contudo, apresentavam referências ou citações de autores que abordavam o ensino da matemática com fractais. De todo modo, não atendiam à temática desta nossa pesquisa e foram desconsiderados durante as análises. Sendo assim, trabalhamos ao todo com 36 trabalhos.

Para a análise dos dados, utilizamos a metodologia de Análise Textual Discursiva. Medeiros e Amorim (2017) destacam que dois importantes elementos devem ser considerados nesse método: categorizar os dados e interpretá-los por meio dos discursos que permeiam a pesquisa. Segundo os autores,

Categorizar é uma das fases da Análise Textual Discursiva, mas assim como na Análise do Discurso, a ênfase se sobressai no discurso. Ela tende a perceber seus objetos de pesquisa como discursos, não como fenômenos isolados. Com isso, registra sua opção de focalizar no todo, concebido como discursos (re)construídos coletivamente. (MEDEIROS; AMORIM, 2017, p. 253).

Inicialmente, foi realizada a categorização dos trabalhos por meio da leitura dos resumos e da busca por palavras-chave, tais como "definição", "característica", "propriedades", 
"irregularidade", “autossemelhança", "complexidade", "dimensão" e "fractais". O intuito foi o de identificar se os autores definiam os fractais usando termos e expressões como "irregularidade", "autossemelhança", "complexidade infinita" e "dimensão fracionária" ou ainda outras nomenclaturas. Em seguida, analisamos o discurso dos autores, com o objetivo de interpretar as suas concepções sobre essas definições.

\section{RESULTADOS E DISCUSSÕES}

Ao analisar os trabalhos, as dificuldades dos autores em conceituar os fractais ficaram evidentes. Da mesma forma como encontramos divergências conceituais sobre o que são tais estruturas em revisões de literatura já aqui mencionadas, os autores ora analisados também fazem alusão a tais divergências. Nos excertos a seguir, destacamos algumas das inquietações encontradas a esse respeito.

\footnotetext{
A definição de Fractal é um problema ainda em aberto na matemática. (TITONELI, 2017, p. 63).

A maior prova da diversidade da Geometria Fractal resume-se no fato de que se quer há uma definição formal, que seja consensual e definitiva do que venha ser um fractal (EBERSON, 2004, p. 16).

Não há uma definição exata e pronta para fractais. (BRISANTE, 2013, p. 16).

$\mathrm{O}$ fato é que, uma definição que conceitue de forma precisa um fractal ainda não foi estabelecida. (MINGORANCI, 2014, p. 33).

Existe uma grande dificuldade de condensar em uma definição matematicamente precisa o que seria um fractal. (BARBOSA, 2019, p. 28).
}

Em 18 dos 36 trabalhos analisados, foi possível detectar angústias similares a essas dos cinco excertos aqui transcritos. Os autores apresentam as divergências encontradas em suas pesquisas introdutórias sobre as definições de "fractal" e mencionam a falta de termos para descrever quais são as características determinantes para tais estruturas. Apesar dessas dificultadas e das divergências encontradas, são mencionadas algumas descrições que se reportam às características mais usadas para conceituar os fractais. Buscamos nos ater a elas.

Como termos e expressões utilizados para se caracterizar tais estruturas, encontramos: "irregularidade", "fractalidade", "falta de escala bem definida", "autossemelhança", "autossimilaridade", "auto-similaridade", "complexidade infinita", "complexidade", “iteração", "iteração infinita", "processo iterativo", "dimensão", "dimensão fractal”, "dimensão de similaridade", "dimensão não inteira", "dimensão fracionária", "dimensionalidade", “dimensão de Hausdorff-Besicovitch" e "dimensão diferente da topológica e Euclidiana". Na figura 1, buscamos sintetizar as categorias que utilizamos para a análise dos trabalhos. 


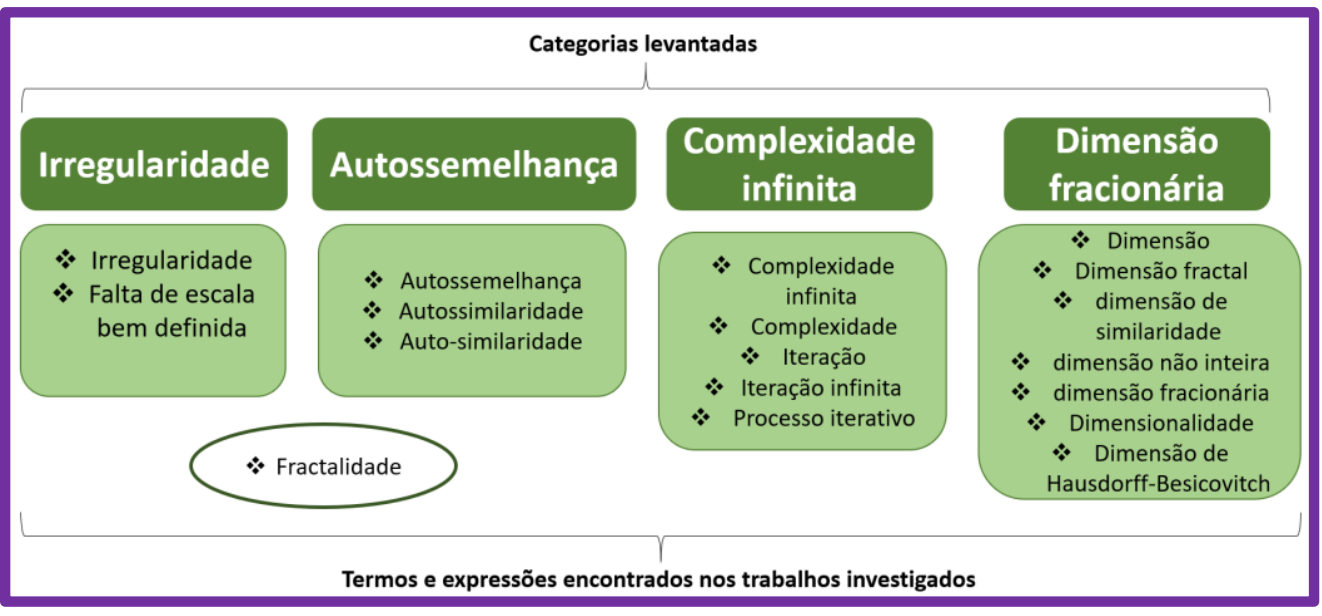

Figura 1 - Sintetização das categorias levantadas frente aos termos encontrados nos trabalhos analisados. Fonte: Elaborado pelos autores (2020).

Entre os termos e expressões encontrados nos trabalhos investigados, mencionados na figura 1, e aqueles que levantamos como sendo hipóteses de categorias relevantes ("irregularidade", "autossemelhança", "complexidade infinita” e "dimensão fracionária”), os termos e expressões que menos se aproximaram das categorias levantadas foram: a fractalidade, apresentada por Santos (2016), e a falta de escala bem definida, referida por Alves (2019). Santos (2016, p. 59) comenta que "fractalidade é uma característica amplamente encontrada na natureza" e que, portanto, faz parte da “definição informal de fractal”. Já Alves (2019, p. 30) refere que a "autossimilaridade" e a "falta de escala bem definida" são "propriedades dos fractais". Com base no contexto apresentado nesses dois trabalhos, supomos que, em ambos os casos, essas categorias remetem à característica da irregularidade. Contudo, tomamos apenas a expressão "falta de escala bem definida" como irregularidade, pois entendemos que, quando temos uma estrutura com a falta de escala bem definida, temos, na verdade, a representação de uma estrutura irregular, que apresenta irregularidades em sua forma.

No quadro 2, apresentamos os resultados encontrados nos 36 trabalhos analisados em relação às características que definem os fractais, expressas pelos autores ou por nós interpretadas. Foram consideradas as categorias "irregularidade", "autossemelhança", "complexidade infinita" e "dimensão fracionária". Para cada trabalho, indicamos a inclusão da característica por "Sim", a não inclusão por "Não" e a não conceituação por parte do autor ou a não identificação por "NC" ("Não consta"). 


\begin{tabular}{|c|c|c|c|c|}
\hline $\begin{array}{l}\text { Autores e (ano de } \\
\text { publicação) }\end{array}$ & Irregularidade & Autossemelhança & $\begin{array}{l}\text { Complexidade } \\
\text { infinita }\end{array}$ & $\begin{array}{c}\text { Dimensão } \\
\text { fracionária }\end{array}$ \\
\hline Eberson (2004) & Não & Sim & Sim & Sim \\
\hline Baier (2005) & Sim & Sim & Não & Sim \\
\hline Gouvea (2005) & Não & Sim & Sim & Sim \\
\hline Gonçalves (2007) & Não & Sim & Não & Sim \\
\hline Stormowski (2008) & $\mathrm{NC}$ & $\mathrm{NC}$ & $\mathrm{NC}$ & $\mathrm{NC}$ \\
\hline Baldovinotti (2011) & Não & Sim & Sim & Sim \\
\hline Krindges (2012) & Não & Sim & Não & Sim \\
\hline Faria (2012) & Não & Sim & Sim & Sim \\
\hline Mineli (2012) & Não & Sim & Sim & Sim \\
\hline Padilha (2012) & Não & Sim & Não & Sim \\
\hline Adami (2013) & Não & Sim & Sim & Sim \\
\hline Brisante (2013) & Não & Sim & Sim & Sim \\
\hline Pereira (2013) & Não & Sim & Sim & Sim \\
\hline Rabay (2013) & Sim & Sim & Sim & Sim \\
\hline Zambelli (2013) & Não & Sim & Sim & Sim \\
\hline Araújo (2014) & Sim & Sim & Sim & Sim \\
\hline Freitas (2014) & Não & Sim & Sim & Sim \\
\hline Mingoranci (2014) & Não & Sim & Sim & Sim \\
\hline Moraes (2014) & Não & Sim & Sim & Sim \\
\hline Nicola (2014) & Não & Sim & Não & Não \\
\hline Paixão (2014) & Não & Sim & Sim & Sim \\
\hline Souza (2014) & Não & Sim & Sim & Sim \\
\hline Andrade Júnior (2015) & Não & Sim & Sim & Sim \\
\hline Araújo (2015) & Não & Sim & Sim & Sim \\
\hline Modesto (2015) & Não & Sim & Sim & Sim \\
\hline Luz (2016) & Não & Sim & Sim & Sim \\
\hline Santos (2016) & Não & Sim & Sim & Sim \\
\hline Mendes Junior (2017) & Sim & Sim & Sim & Sim \\
\hline Moreira (2017) & Sim & Sim & Sim & Não \\
\hline Santos (2017) & $\mathrm{NC}$ & $\mathrm{NC}$ & $\mathrm{NC}$ & $\mathrm{NC}$ \\
\hline Titoneli (2017) & Não & Sim & Sim & Sim \\
\hline Duarte (2018) & $\mathrm{NC}$ & $\mathrm{NC}$ & $\mathrm{NC}$ & $\mathrm{NC}$ \\
\hline Valmorbida (2018) & Não & Sim & Sim & Sim \\
\hline Alves (2019) & Sim & Sim & Sim & Não \\
\hline Barbosa (2019) & Não & Sim & Sim & Sim \\
\hline Oliveira (2019) & Não & Sim & Sim & Sim \\
\hline Vieira (2019) & Não & Sim & Sim & Sim \\
\hline
\end{tabular}

Quadro 2 - Presença as características "irregularidade", "autossemelhança”, "complexidade infinita" e "dimensão fracionária" nos trabalhos analisados.

Fonte: Dados da pesquisa (2020).

A partir do quadro 2, percebe-se que as características "irregularidade", "autossemelhança", "complexidade infinita" e "dimensão fracionária" marcaram presença como características que definem os fractais. A irregularidade foi a que menos se destacou, sendo indicada por apenas seis dos 36 trabalhos analisados. Destacamos, contudo, que, em 21 trabalhos, o termo foi utilizado não como uma característica que contribui para a definição dos fractais, mas como explicação para a adoção do substantivo "fractal" - como mencionado na 
fundamentação deste artigo, devido à sua raiz etimológica, esse termo, por si só, faz menção à irregularidade.

Além disso, em 14 trabalhos, os autores associaram a irregularidade à ideia de dimensão.

De fato, o cálculo da dimensão fractal consegue medir o "grau de irregularidade" ou o "nível de irregularidade" de um fractal. Porém, vale ressaltar que a irregularidade e a dimensão fracionária são características distintas. A dimensão fracionária é a característica que quantifica de forma fracionária a irregularidade de um fractal; já a irregularidade é a característica que está sendo quantificada de forma fracionária. Nos excertos a seguir, apresentamos algumas das formas como a irregularidade apareceu nos trabalhos:

\footnotetext{
A Geometria Fractal de Mandelbrot reflete uma natureza de irregularidade, de reentrâncias, saliências e representações, de fragmentações [...]. (GONÇALVES, 2007, p. 40).

As irregularidades ali destacadas também foram percebidas em diversos fenômenos naturais, como relâmpagos, formato de montanhas [...]. (PADILHA, 2012, p. 33).

Os padrões encontrados na natureza são tão irregulares e fragmentados, que a Geometria tradicional não é capaz de descrever de forma satisfatória. (ANDRADE JÚNIOR, 2015, p. 45).

[Definição] introduzida por Mandelbrot, que tem origem no adjetivo em latim fractus, que significa irregular ou quebrado. (LUZ, 2016, p. 25).

Os fractais apresentam um grau de irregularidade em qualquer escala. (MENDES JÚNIOR, 2017, p. 22).

A dimensão fractal apresenta o nível de irregularidade. (BARBOSA, 2019, p. 28).
}

Com base nesses excertos, surgem as seguintes questões: será que a irregularidade é uma das características que definem os fractais, visto que a "Geometria Fractal de Mandelbrot reflete uma natureza de irregularidade"? (GONÇALVES, 2007, p. 40). A “irregularidade em qualquer escala" não é uma característica dos fractais? (MENDES JÚNIOR, 2017, p. 22). E se o termo "fractal" remete a algo "irregular", por que não considerar esse termo como uma característica que explica/define tal estrutura? (LUZ, 2016, p. 25). Portanto, para nós, a irregularidade é, sim, uma característica que contribui para a definição dos fractais.

Ainda sobre a irregularidade, observemos a figura 2. Nela, temos um quadrado que foi subdividido em partes proporcionais. Admitindo que esse processo de divisão seja infinito, temos nessa estrutura (o quadrado) uma das características dos fractais - a da complexidade infinita. 


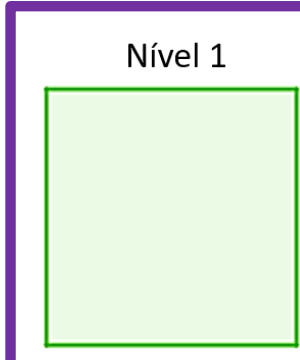

№ de quadrados 1
Nível 2

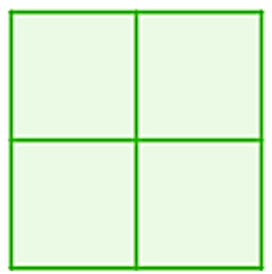

№ de quadrados 4
Nível 3

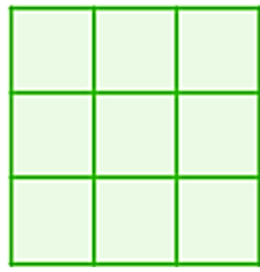

№ de quadrados 9
Nível 4

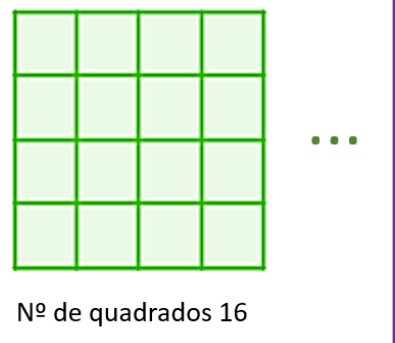

Figura 2 - Quadrados divididos em lados proporcionais. Fonte: Adaptado de Rabay (2013).

Se calcularmos a dimensão fracionária para a figura da ilustração, observando o nível ${ }^{3}$ 2 e utilizando a fórmula $D=\frac{\log n}{\log r}$, em que $n=4$ (pois indica o número partes geradas no "fractal" (Quadrado)) e $r=\frac{1}{1 / 2}$, a medida o lado no nível $2=2$. Assim, teremos: $D=\frac{\log 4}{\log 2}=$ 2. Logo, temos que essa estrutura (o quadrado), divido infinitamente em processos recursivos, apresenta complexidade infinita e dimensão fracionária. Além disso, intuitivamente podemos constatar que ele apresenta autossemelhança, pois seus quadrados são semelhantes entre si. Então, é correto afirmar que essa estrutura é um fractal? Não, pois falta-lhe a característica da irregularidade.

$\mathrm{Na}$ figura 3, realizamos os mesmos procedimentos anteriores, mas desta feita observando o fractal do triângulo de Sierpinski, um fractal clássico do tipo remoção, em que, a cada nível, são retirados triângulos semelhantes de seu interior. Vejamos as características da figura 3 .

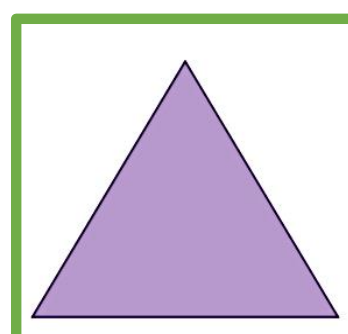

Nível 0

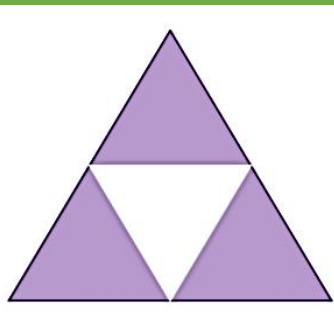

Nível 1

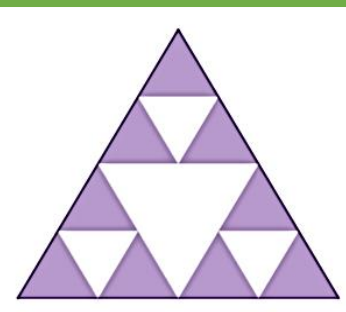

Nível 2

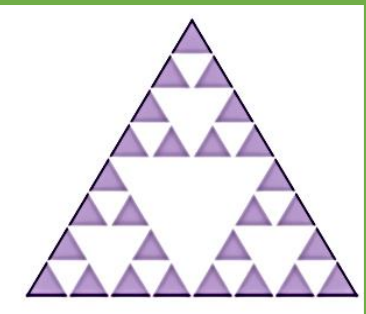

Nível 3

Figura 3 - Alguns níveis do triângulo de Sierpinski. Fonte: Elaborado pelos autores.

A figura 3 também ilustra uma estrutura em que continuamente se verifica o processo de recursividade infinita; logo, existe nesse triângulo a característica da complexidade infinita.

${ }^{3}$ Poderíamos escolher qualquer nível, a não ser o primeiro, pois $n$ e $r$ não podem ser iguais a 1. 
Por mais que se retirem triângulos do interior da figura, a estrutura preserva características de semelhança - então, podemos afirmar que ela apresenta a característica de autossemelhança. Além disso, existe a dimensão fracionária, que pode ser calculada observando que o nível 1 é dado por $D=\frac{\log 3}{\log 2} \cong 1,58$, pois $n=3$. O número de peças pintadas no nível 1 e $r=\frac{1}{1 / 2}$, pois o lado de cada triângulo pintado nesse nível é $\frac{1}{2}$. Existe ainda a irregularidade, que pode ser identificada intuitivamente pelos "furos" em formato de triângulos, em branco, retirados a cada nível.

Em outras palavras, na figura 2, temos um Quadrado, divido e repetido infinitamente, mas que continua representando uma forma geométrica euclidiana. Já na figura 3, ali sim, temos um fractal, conhecido como o "Fractal do triângulo de Sierpinski". Perceba que a única diferença entre as duas estruturas é a irregularidade e que as demais características investigadas e apontadas neste artigo foram suficientes para definir e distinguir ambas as formas. Diante disso, não só consideramos a irregularidade como válida e fundamental para definir os fractais, como também indicamos que ela seja a primeira característica avaliada ao se investigar tais estruturas.

A partir do que foi exposto, em resumo, temos que os fractais podem serem considerados como formas ou estruturas geométricas com as características de irregularidade, autossemelhança, complexidade infinita e dimensão fracionária. Cada uma delas contribui de forma autônoma para a definição da estrutura em questão.

A irregularidade é a característica que remete às partes irregulares de uma estrutura fractal, podendo ser percebida como estruturas rugosas, furos, partes com tamanhos e formas diferentes ou "quebradas". A autossemelhança é a característica relacionada com o padrão que essas formas apresentam em sua estrutura - ou seja, é possível perceber partes (formas geométricas, como segmentos, curvas, polígonos, estruturas tridimensionais etc.) se repetindo de forma semelhante umas em relação às outras, a cada nível (iteração, passo recursivo). Já a complexidade infinita é a característica relacionada à recursividade. Geralmente, essa recursividade é infinita, mas, principalmente em casos de objetos, ela pode ser finita. Por último, a dimensão fracionária é aquela que calcula o grau de irregularidade de um fractal, encontrando-se entre os valores topológicos $0,1,2$ ou 3. 


\section{CONSIDERAÇÕES FINAIS}

Este artigo buscou investigar o que são os fractais e quais são as características que os definem a partir de pesquisas brasileiras. Durante a realização de uma pesquisa de mestrado, percebemos divergências conceituais em diversos trabalhos que abordavam esse tema. Este artigo surgiu a partir da compreensão de que definir corretamente tais estruturas, principalmente em trabalhos científicos, é de fundamental importância para que o ensino da Geometria Fractal venha a ser implementada com maior efetividade nos contextos educacionais. Com a análise de 36 trabalhos de pesquisas encontradas na BDTD, este artigo objetivou lançar uma contribuição para as discussões acerca desta problemática.

Entre os trabalhos analisados a respeito do ensino da matemática com fractais, apenas um correspondeu a uma pesquisa de doutorado sobre o tema. Em 18 relatos dos 36 trabalhos analisados, foi possível encontrar divergências ou dificuldades dos autores de se definir os fractais. Esses dados apontam para a necessidade de pesquisas mais aprofundas sobre o tema no contexto científico brasileiro. Os impasses em relação à definição dos fractais podem ser um dos fatores que influenciam a ausência da sua abordagem no contexto de Ensino Básico brasileiro. Vale ressaltar que uma das preocupações dos pesquisadores que tratam do ensino da matemática com os fractais é que estes saberes se tornem tão acessíveis a ponto de serem incorporados a práticas efetivas de ensino. Consideramos que o ponto de partida para romper essa barreira que impede a popularização dessa geometria é buscar conhecer mais sobre o assunto e discutir as concepções preestabelecidas sobre o tema.

Dentre os resultados alcançados neste trabalho que contribuem para a compreensão do que são os fractais, no que diz respeito a seus elementos conceituais, destacamos as características de irregularidade, autossemelhança, complexidade infinita e dimensão fracionária como sendo as identificadas nos trabalhos analisados para definir os fractais. Além dessas características que definem tais estruturas, a designação do que elas são também é um tópico importante para futuros trabalhos.

Nas pesquisas analisadas, encontramos diferentes termos para fazer menção anafórica aos fractais - "objetos", "figuras", "formas" e "estruturas". A partir desses usos, entendemos que os fractais devem ser referidos como "formas" ou "estruturas geométricas", pois representam situações físicas e abstratas presentes nas interações humanas. De todo modo, sugerimos que esse tópico da definição/designação dos fractais deve ser ainda objeto de reflexão científica e de discussão em outros trabalhos. 
Com base no exposto, espera-se que este artigo venha a contribuir para o avanço da definição dos fractais, para melhor se compreender o que são tais estruturas geométricas e para fazer com que essa geometria seja conhecida principalmente no contexto do Ensino Básico brasileiro, etapa na qual se verifica a necessidade de uma disseminação desse conhecimento.

\section{REFERÊNCIAS}

ADAMI, P. S. Fractais no Ensino Médio: uma sequência didática. 2013. 55 f. Dissertação (Mestrado em Ciências Exatas e da Terra) - Universidade Federal de São Carlos, São Carlos, 2013. Disponível em: https://bit.ly/36sT5FL. Acesso em: 20 de jan. 2020.

ANDRADE JÚNIOR, E. A. de. Ciência contemporânea a formação de professores: o caso dos fractais em uma perspectiva Kelliana. 2015. 162 f. Dissertação (Programa de PósGraduação em Ensino das Ciências) - Universidade Federal Rural de Pernambuco, Recife. Disponível em: https://bit.ly/3h3cKCW. Acesso em: 20 de jan. 2020.

ALVES, D. C. S. Fractais: uma ferramenta no Ensino Médio. 2014. 80 f. Dissertação (Mestrado Profissional em Matemática em Rede Nacional) - Departamento de Matemática, Universidade Federal de Sergipe, São Cristóvão, 2014. Disponível em: https://bit.ly/2UhSIKX. Acesso em: 20 de jan. 2020.

ARAÚJO, A. T. G. de Noções de Geometria Fractal elementar. 2014. 68 f. Dissertação (Mestrado Profissional em Matemática em Rede Nacional) - Departamento de Matemática, Universidade Federal de Sergipe, São Cristóvão, 2014. Disponível em: https://bit.ly/3gWUZ85. Acesso em: 20 de jan. 2020.

ARAÚJO, J. M. Teoria matemática implícita na Geometria Fractal: construindo fractais com a ferramenta computacional Asypmtote. 2015. 69 f. Dissertação (Mestrado Profissional em Matemática em Rede Nacional) - Universidade Federal de Roraima, Boa Vista, 2015. Disponível em: https://bit.ly/3gWV8Zb. Acesso em: 20 de jan. 2020.

BAIER, T. O nexo "Geometria Fractal - produção da ciência contemporânea" tomado como núcleo do currículo do Ensino Básico. 2005. 147 f. Tese (Doutorado em Educação Matemática) - Instituto de Geociências e Ciências Exatas, Universidade Estadual Paulista, Rio Claro, 2005. Disponível em: https://bit.ly/2GqDgon. Acesso em: 20 jan. 2020.

BALDOVINOTTI, N. J. Um estudo de fractais geométricos na formação de professores de matemática. 2011. 204 f. Dissertação (Mestrado em Educação Matemática) Instituto de Geociências e Ciências Exatas, Universidade Estadual Paulista, Rio Claro, 2011. Disponível em: https://bit.ly/2A9EWDf. Acesso em: 20 jan. 2020.

BRISANTE, I. M. Fractais no Ensino Médio. 2013. 44 f. Dissertação (Profissional de Matemática em Rede Nacional) - Instituto de Biociências, Letras e Ciências Exatas, Universidade Estadual Paulista, São José do Rio Preto, 2013. Disponível em: https://bit.ly/2Y4KYNz. Acesso em: 20 jan. 2020. 
BAPTISTA, T. R. F. Fractais - aplicações em Engenharia. 2013. 76 f. Dissertação

(Mestrado em Engenharia Electrotécnica e de Computadores) - Departamento de Engenharia Electrotécnica, Instituto Superior de Engenharia do Porto, Porto, Portugal, 2013. Disponível em: https://bit.ly/3097kQG. Acesso em: 20 jan. 2020.

BARBOSA, L. M. Aspetos do pensamento computacional na construção de fractais com software GeoGebra. 2019. 169 f. Dissertação (Mestrado em Educação Matemática) Instituto de Geociências e Ciências Exatas, Universidade Estadual Paulista, Rio Claro, 2019. Disponível em: https://bit.ly/2z8Ncmx. Acesso em: 20 jan. 2020.

BARBOSA, R. M. Descobrindo a Geometria Fractal - para a sala de aula. 3. ed. Belo Horizonte: Autêntica, 2005.

BONI, G. E. O.; ALENCAR E. S. O Currículo para o Ensino De Matemática na Formação de Professoras Normalistas em Colégios Confessionais Católicos do Sul de Mato Grosso (19591963). Reamec - Rede Amazônica de Educação em Ciências e Matemática, v. 7, n. 3, Cuiabá, 2019.

CAMPOS, F. A. B.; FAGUNDES, M. C. Os Fractais que Nos Rodeiam. In: I Encontro MatoGrossense de Professores que Ensinam Matemática - I Emapem. Anais [...], Tangará da Serra, Mato Grosso, 2018. Disponível em: https://bit.ly/37tf54J. Acesso em: 20 jan. 2020.

DALPIAZ, M. R. Um estudo sobre fractais: origem e proposta didática para aplicação em aula. 2016. 72 f. Dissertação (Mestrado Profissional em Matemática em Rede Nacional) Universidade Tecnológica Federal do Paraná, Curitiba, 2016. Disponível em:

https://bit.ly/2u3iZTt. Acesso em: 20 jan. 2020.

DUARTE, R. C. B. C. Utilização do GeoGebra, de smartphone e de reflexões escritas na construção de conceitos relacionados a retas paralelas cortadas por uma transversal. 2018. 123 f. Dissertação (Mestrado Profissional em Educação em Ciências Matemática) Instituto de Educação, Universidade Federal Rural do Rio de Janeiro, Seropédica, 2018. Disponível em: https://bit.ly/30av0El. Acesso em: 20 jan. 2020.

EBERSON, R. R. Um estudo sobre a construção de fractais em ambientes computacionais e suas relações como transformações geométricas no plano. 2004. $177 \mathrm{f}$. Dissertação (Mestrado em Educação) - Pontifícia Universidade Católica de São Paulo, São Paulo, 2004. Disponível em: https://bit.ly/2A9Gi0N. Acesso em: 20 jan. 2020.

FALCONER, K. J. Fractal Geometry: mathematical foundations and applications. New York: John Wiley \& Sons, 1985.

FARIA, R. W. S. Padrões fractais: contribuições ao processo de generalização de conteúdos matemáticos. 2012. 197 f. Dissertação (Mestrado em Educação Matemática) - Instituto de Geociências e Ciências Exatas, Universidade Estadual Paulista, Rio Claro, 2012. Disponível em: https://bit.ly/2S2F78k. Acesso em: 20 jan. 2020.

FREITAS, A. F. Explorando o conjunto de Cantor e outros fractais no Ensino Básico. 2014. 44 f. Dissertação (Mestrado em Educação Matemática) - Instituto de Biociências, Ciências Humanas e Ciências Exatas, Universidade Estadual Paulista, 2014. Disponível em: https://bit.ly/2A5bs9y. Acesso em: 20 jan. 2020. 
GERHARDT, T. E.; SILVEIRA, D. T. (org.) Método de pesquisa. Porto Alegre: Ed. da UFRGS, 2009.

GONÇALVES, A. G. N. Uma sequência de ensino para o estudo de progressões geométricas via fractais. 2007. 215 f. Dissertação (Mestrado em Educação) - Pontifícia Universidade Católica de São Paulo, São Paulo, 2007. Disponível em: https://bit.ly/37a17oX. Acesso em: 20 jan. 2020.

GOUVEA, F. R. Um estudo de fractais geométricos através de caleidoscópios e softwares de geometria dinâmica. 2005. 259 f. Dissertação (Mestrado em Educação Matemática) Instituto de Geociências e Ciências Exatas, Universidade Estadual Paulista, Rio Claro, 2005. Disponível em: https://bit.ly/2OaDAvT. Acesso em: 20 jan. 2020.

KINDGES, E. E. Geometria Fractal no Ensino Fundamental: inserindo matemática contemporânea nos conteúdos do currículo escolar. 2012. 94 f. Dissertação (Mestrado em Ensino de Ciências Naturais e Matemática) - Centro de Ciências Exatas e Naturais, Universidade Regional de Blumenau, Blumenau, 2012. Disponível em: https://bit.ly/2Mx Y94d. Acesso em: 20 jan. 2020.

LUZ, E. V. A Geometria Fractal como fator minimizador das dificuldades referentes a conceitos geométricos. 2016. 83 f. Dissertação (Mestrado Profissional em Matemática em Rede Nacional) - Instituto de Biociências, Ciências Humanas e Ciências Exatas, Universidade Estadual Paulista, São José do Rio Preto, 2016. Disponível em: https://bit.ly/2z8P6Ud. Acesso em: 20 jan. 2020.

LÜDKE, M.; ANDRÉ, M. E. D. A. Pesquisas em Educação: abordagens qualitativas. São Paulo: EPU, 1986.

MANDELBROT, B. B. The Fractal Geometry of Nature. New York: Freeman, 1977.

MENDES JÚNIOR, D. de C. Uma nova abordagem dos complexos para o Ensino Médio: o estudo dos fractais e do caos na composição do conjunto preenchido de Julia e o conjunto de Mandelbrot. 2017. 109 f. Dissertação (Mestrado Profissional em Matemática em Rede Nacional) - Centro de Matemática, Computação e Cognição, Universidade Federal do ABC, Santo André, 2017. Disponível em: https://bit.ly/38YiXv0. Acesso em: 20 jan. 2020.

MINGORANCI, S. A Geometria Fractal aliada à contextualização, protagonismo juvenil e tecnologias como proposta de melhoria no processo ensino/aprendizagem da matemática na Educação Básica. 2014. 121 f. Dissertação (Mestrado Profissional em Matemática em Rede Nacional) - Universidade Federal de Mato Grosso do Sul, Três Lagos, 2014. Disponível em: https://bit.ly/3eWXs0A. Acesso em: 20 jan. 2020.

MODESTO, C. F. Matemática e arte: explorando a geometria dos fractais e as tesselações de Escher. 2015. 120 f. Dissertação (Mestrado em Matemática) - Centro de Ciências Exatas, Universidade Estadual de Londrina, Londrina, 2015. Disponível em: https://bit.ly/2UdfYKg. Acesso em: 20 jan. 2020.

MORAES, L. Equações de diferenças, caos e fractais. 2014. 117 f. Dissertação (Mestrado Profissional em Matemática em Rede Nacional) - Centro de Ciências Físicas e Matemática, 
Universidade Federal de Santa Catarina, Florianópolis, 2014. Disponível em: https://bit.ly/2MzAu3o. Acesso em: 20 jan. 2020.

MOREIRA, V. S. S. S. Geometria Fractal na Educação Básica. 2017. 78 f. Dissertação (Mestrado Profissional em Matemática em Rede Nacional) - Departamento de Matemática, Pontifícia Universidade Católica do Rio de Janeiro, Rio de Janeiro, 2017. Disponível em: https://bit.ly/2BE3xka. Acesso em: 20 jan. 2020.

MEDEIROS, E. A.; AMORIM, G. C. C. Análise textual discursiva: dispositivo analítico de dados qualitativos para a pesquisa em educação. Laplage em Revista, Sorocaba, v. 3, n. 3, p. 247-260, 2017.

MINELI, J. P. Fractais: generalização de padrões no Ensino Fundamental. 2012. 88 f. Dissertação (Mestrado em Educação) - Departamento de Matemática, Pontifícia Universidade Católica de São Paulo, São Paulo, 2012. Disponível em: https://bit.ly/2AZeSL5. Acesso em: 20 jan. 2020.

MUCHERONI, L. F. Dimensão de Haudorff e algumas aplicações. 2017. 63 f. Dissertação (Mestrado em Matemática) - Instituto de Geociências e Ciências Exatas, Universidade Estadual Paulista, Rio Claro, 2017. Disponível em: https://bit.ly/2ATtcVG. Acesso em: 20 jan. 2020.

NICOLA, C. H. Conhecendo fractal no Ensino Médio: árvore pitagórica. 2013. 63 f. Dissertação (Mestrado Profissional em Matemática em Rede Nacional) - Departamento de Matemática, Universidade Federal de São Carlos, São Carlos, 2014. Disponível em: https://bit.ly/2MH1fmF. Acesso em: 20 jan. 2020.

NEGRI, M. G. Introdução ao Estudo dos Fractais. 2014. 60 f. Dissertação (Mestrado Profissional em Matemática em Rede Nacional) - Universidade Federal de Goiás, Goiânia, 2014. Disponível em: https://bit.ly/37111JZ. Acesso em: 20 jan. 2020.

NUNES, R. S. R. Geometria Fractal e aplicações. 2006. 78 f. Dissertação (Mestrado em Ensino da Matemática) - Departamento de Matemática Pura, Faculdade de Ciências, Universidade do Porto, Porto, 2006. Disponível em: https://bit.ly/2Yy8anN. Acesso em: 20 jan. 2020.

OLIVEIRA, L. M. Uma proposta de Geometria de Fractais para a sala de aula. 2019. 41 f. Dissertação (Mestrado Profissional em Matemática em Rede Nacional) - Instituto de Ciências Exatas, Universidade Federal de Juiz de Fora, Juiz de Fora, 2019. Disponível em: https://bit.ly/37fPYTG. Acesso em: 20 jan. 2020.

PADILHA, T. A. F. Conhecimentos geométricos e algébricos a partir da construção de fractais com uso do software GeoGebra. 2012. 140 f. Dissertação (Mestrado Profissional em Ensino de Ciências Exatas) - Centro Universitário Univates, Lajeado, 2012. Disponível em: https://bit.ly/2YkzlSH. Acesso em: 20 jan. 2020.

PAIXÃO, R. S. O ensino de fractais no Ensino Fundamental. 2013. 68 f. Dissertação (Mestrado Profissional em Matemática em Rede Nacional) - Departamento de Matemática, Universidade Estadual de Maringá, Maringá, 2013. Disponível em: https://bit.ly/3hencr2. Acesso em: 20 jan. 2020. 
PEREIRA, A. S. Fractais circulares: algumas considerações e atividades. 2013. $81 \mathrm{f}$. Dissertação (Mestrado Profissional em Matemática em Rede Nacional) - Centro de Ciências Exatas, Universidade Estadual de Londrina, Londrina, 2013. Disponível em: https://bit.ly/30sXEk6. Acesso em: 20 jan. 2020.

RABAY, Y. S. F. Estudo e aplicações da Geometria Fractal. 2013. 103 f. Dissertação (Mestrado Profissional em Matemática em Rede Nacional) - Centro de Ciências Exatas e da Natureza, Departamento de Matemática, Universidade Federal da Paraíba, João Pessoa, 2013. Disponível em: https://bit.ly/2Yqc0iK. Acesso em: 20 jan. 2020.

SANTOS, C. X. M. Circuitos resistivos autossimilares. 2016. 103 f. Dissertação (Mestrado Profissional em Matemática em Rede Nacional) - Instituto de Ciências Matemáticas e Computação, Universidade de São Paulo, São Carlos, 2016. Disponível em: https://bit.ly/2YnIWrS. Acesso em: 20 jan. 2020.

SANTOS, N. L. P. S. O misterioso e enigmático mundo de Pascal e Fibonacci. 2017. 113 f. Dissertação (Mestrado em Educação Matemática) - Instituto de Biociências, Letras e Ciências Exatas, Universidade Estadual Paulista, São José do Rio Preto, 2017. Disponível em: https://bit.ly/2AnS3kv. Acesso em: 20 jan. 2020.

SÁ-SILVA, J. R.; ALMEIDA, C. D.; GUINDANI, J. P. Pesquisa Documental: pistas teóricas e metodológicas. Revista Brasileira de História \& Ciências Sociais, ano 1, n 1, p. 1-15, 2009.

SOUZA, C. Geometria Fractal e aplicações no Ensino Médio. 2014. 74 f. Dissertação (Mestrado em Matemática) - Instituto de Ciências Exatas, Departamento de Matemática, Universidade de Brasília, Brasília, 2014. Disponível em: https://bit.ly/3dRtueb. Acesso em: 20 jan. 2020.

STORMOWSKI, V. Estudando matrizes a partir de transformações geométricas. 2008. 157 f. Dissertação (Mestrado Profissionalizante em Ensino de Matemática) - Universidade Federal do Rio Grande do Sul, Porto Alegre, 2008. Disponível em: https://bit.ly/2MI7Cpx. Acesso em: 20 jan. 2020.

TITONELI, L. M. B. A observação de padrões: modelagem matemática através de sequências numéricas e objetos geométricos. 2017. 78 f. Dissertação (Mestrado em Matemática) - Departamento de Matemática, Pontifícia Universidade Católica do Rio de Janeiro, Rio de Janeiro, 2017. Disponível em: https://bit.ly/2UtkowA. Acesso em: 20 jan. 2020.

TUZZO, S. A.; BRAGA, C. F. O processo de triangulação da pesquisa qualitativa: o metafenômeno como gênese. Revista Pesquisa Qualitativa, v. 4, n. 5, São Paulo, 2016. Disponível em: https://bit.ly/36MAULe. Acesso em: 20 jan. 2020.

VALMORBIDA, J. M. Uma proposta de atividades para o estudo de progressões geométricas utilizando fractais e o software GeoGebra. 2018. 128 f. Dissertação (Mestrado Profissional em Matemática em Rede Nacional) - Universidade Federal Fronteira Sul, Chapecó, 2018. Disponível em: https://bit.ly/2An46hP. Acesso em: 20 jan. 2020. 
VIEIRA, D. C. O uso da Geometria Fractal como Ferramenta no ensino de progressões geométricas e logaritmos. 2019. 90 f. Dissertação (Mestrado Profissional em Ensino de Ciências Exatas) - Departamento de Matemática, Universidade Federal de São Carlos, São Carlos, 2019. Disponível em: https://bit.ly/3f9CewC. Acesso em: 20 jan. 2020.

VIEIRA, L. B. Implicações pedagógicas do lúdico para o ensino e aprendizagem da álgebra. 2011. 125 f. Dissertação (Mestrado em Educação em Ciências e Matemática) Universidade Federal de Goiás, Goiânia, 2011. Disponível em: https://bit.ly/30sYq0G. Acesso em: 20 jan. 2020.

ZAMBELLI, J. A. G. J. Fractais no Ensino Médio: a valorização da geometria a partir de uma nova experiência em sala de aula. 2013. 87 f. Dissertação (Mestrado Profissional em Matemática em Rede Nacional) - Universidade Federal do ABC, Santo André, 2013. Disponível em: https://bit.ly/3cI7EbK. Acesso em: 20 jan. 2020.

Submetido em: 08 de abril de 2020 .

Aprovado em: 21 de maio de 2020. 